\title{
PARTITIONED TRANSVERSALS
}

\author{
by L. MIRSKY
}

(Received 8 May, 1972)

Let $E$ be a finite set and let $\mathfrak{A}=\left(A_{1}, \ldots, A_{n}\right)$ be a family of subsets of $E$. A subset $T$ of $E$ is called a transversal of $\mathfrak{U}$ if there exists a bijection $\psi: T \rightarrow\{1, \ldots, n\}$ such that $x \in A_{\psi(x)}$ for all $x \in \mathrm{T}$. If $\mathrm{I} \subseteq\{1, \ldots, n\}$, we shall, for brevity, write

$$
\mathrm{A}(\mathrm{I})=\bigcup_{i \in I} \mathrm{~A}_{i}
$$

(and similarly for families denoted by other letters). The cardinal of a set $\mathrm{S}$ will be denoted by $|S|$. If $\lambda$ is a non-negative integer, we define $\lambda S$ as $S$ or $\emptyset$ according as $\lambda>0$ or $\lambda=0$.

A criterion for the existence of transversals was established in P. Hall's celebrated paper [2] which appeared in 1935.

THeOREM 1. The family $\mathfrak{U}=\left(\mathrm{A}_{1}, \ldots, \mathrm{A}_{n}\right)$ of sets possesses a transversal if and only if $|\mathrm{A}(\mathrm{I})| \geqq|\mathrm{I}|$ for all $\mathrm{I} \subseteq\{1, \ldots, n\}$.

Making use of the duality theorem of linear programming, Hoffman and Kuhn [3] obtained in 1956 the following striking generalization of Hall's criterion.

THEOREM 2. Let $\left(\mathrm{E}_{1}, \ldots, \mathrm{E}_{p}\right)$ be a partition (into pairwise disjoint subsets) of a finite set $\mathrm{E}$; let $\mathfrak{A}=\left(\mathrm{A}_{1}, \ldots, \mathrm{A}_{n}\right)$ be a family of subsets of $\mathrm{E}$; and let $0 \leqq r_{j} \leqq s_{j}(1 \leqq j \leqq p)$ be integers. Then $\mathfrak{A}$ possesses a transversal, say $\mathrm{T}$, such that

if and only if

$$
r_{j} \leqq\left|\mathrm{~T} \cap \mathrm{E}_{j}\right| \leqq s_{j} \quad(1 \leqq j \leqq p)
$$

for all $\mathrm{I} \subseteq\{1, \ldots, n\}, \mathrm{J} \subseteq\{1, \ldots, p\}$.

$$
|\mathrm{A}(\mathrm{I}) \cap \mathrm{E}(\mathrm{J})| \geqq|\mathrm{I}|-\min \left\{n-\sum_{j \in \mathrm{J}} r_{j}, \sum_{j \in J} s_{j}\right\}
$$

For $p=1, r_{1}=s_{1}=n$ this reduces, of course, to Theorem 1. In addition to the original treatment of Hoffman and Kuhn, there exist several alternative proofs. Most of these make use of ideas drawn from the theory of abstract independence and invoke at some stage Rado's theorem on independent transversals [7] (which is itself a generalization of Theorem 1). In this context, we may mention the proofs of D. J. A. Welsh [8], of Hazel Perfect [6, Chap. VII], and of the present writer [5, Chap. 9]. Another proof is to be found in Ford and Fulkerson's book [1, p. 75], where Theorem 2 is deduced from the " max-flow min-cut" theorem, a result intimately related to Menger's graph theorem. Here we adopt an altogether different approach and give a demonstration of Theorem 2 which presupposes no background other than a knowledge of P. Hall's fundamental criterion. This treatment provides yet a further illustration of the theme of "self-refinement" of Hall's criterion discussed in a recent expository article [4] by the author.

If $\mathfrak{A}$ possesses a transversal $T$ which satisfies (1), then obviously

$$
r_{j} \leqq\left|\mathrm{E}_{j}\right| \quad(1 \leqq j \leqq p), \quad \sum_{j=1}^{p} r_{j} \leqq n \leqq \sum_{j=1}^{p} s_{j} .
$$


But equally, these relations follow if (2) is assumed to hold for all I and J. Thus the validity of (3) can be assumed throughout the argument.

Let $\mathrm{D}_{1}, \ldots, \mathrm{D}_{p}$ be sets such that $\mathrm{E}, \mathrm{D}_{1}, \ldots, \mathrm{D}_{p}$ are pairwise disjoint and $\left|\mathrm{D}_{j}\right|=s_{j}-r_{j}$ $(1 \leqq j \leqq p)$. Denote by $\mathfrak{I}^{*}$ the family of subsets of $E \cup D_{1} \cup \ldots \cup D_{p}$ consisting of $\mathrm{A}_{1}, \ldots, \mathrm{A}_{n}$ together with $\left|\mathrm{E}_{j}\right|-r_{j}$ copies of $\mathrm{E}_{j} \cup \mathrm{D}_{j}(1 \leqq j \leqq p)$ and $\sum_{1}^{p} s_{j}-n$ copies of $\mathrm{D}_{1} \cup \ldots \cup \mathrm{D}_{p}$. We note that the number of sets in $\mathfrak{I}^{*}$ is equal to the cardinal of the ground set, namely

$$
|\mathrm{E}|+\sum_{1}^{p}\left(s_{j}-r_{j}\right) \text {. }
$$

Hence a transversal of $\mathfrak{Q}^{*}$, if one exists, must be identical with the ground set.

Suppose, in the first place, that $\mathfrak{X}^{*}$ has a transversal. Then a certain subset of this transversal, say $T(\subseteq E)$, is a transversal of $\mathfrak{A}$. Write

so that

$$
\left|\mathrm{T} \cap \mathrm{E}_{j}\right|=t_{j} \quad(1 \leqq j \leqq p)
$$

$$
t_{j} \leqq\left|\mathrm{E}_{j}\right| \quad(1 \leqq j \leqq p), \quad \sum_{j=1}^{p} t_{j}=n .
$$

Now $t_{j}$ elements of $E_{j}$ have been used in $T$; hence the $\left|E_{j}\right|-r_{j}$ copies of $E_{j} \cup D_{j}$ are represented by $\left|E_{j}\right|-t_{j}$ elements of $E_{j}$ (so that $\left|E_{j}\right|-t_{j} \leqq\left|E_{j}\right|-r_{j}$ ) and $t_{j}-r_{j}$ elements of $D_{j}$ (so that $t_{j}-r_{j} \leqq s_{j}-r_{j}$ ). Thus $r_{j} \leqq t_{j} \leqq s_{j} ;$ and T satisfies (1).

Conversely, suppose that $\mathfrak{A}$ has a transversal $\mathrm{T}$ which satisfies (1); and use the notation in (4), so that $r_{j} \leqq t_{j} \leqq s_{j}(1 \leqq j \leqq p)$. Let the $\left|E_{j}\right|-r_{j}$ copies of $E_{j} \cup D_{j}$ be represented by the $\left|\mathrm{E}_{j}\right|-t_{j}$ elements of $\mathrm{E}_{j}$ not used in $\mathrm{T}$ and by $t_{j}-r_{j}$ elements of $\mathrm{D}_{j}(1 \leqq j \leqq p)$. We are then left with $s_{j}-t_{j}$ elements of $\mathrm{D}_{j}(1 \leqq j \leqq p)$, and these elements just suffice to represent the $\sum_{1}^{p} s_{j}-n$ copies of $\mathrm{D}_{1} \cup \ldots \cup \mathrm{D}_{p}$, since $\sum_{1}^{p} t_{j}=n$. Hence $\mathfrak{A}^{*}$ has a transversal.

We have thus shown that $\mathfrak{A}$ has a transversal $\mathrm{T}$ subject to (1) if and only if $\mathfrak{A}^{*}$ has a transversal. By Hall's criterion (Theorem 1), this is the case if and only if

$$
\left|\mathrm{A}(\mathrm{I}) \cup \bigcup_{j=1}^{p} \lambda_{j}\left(\mathrm{E}_{j} \cup \mathrm{D}_{j}\right) \cup \mu\left(\mathrm{D}_{1} \cup \ldots \cup \mathrm{D}_{p}\right)\right| \geqq|\mathrm{I}|+\sum_{j=1}^{p} \lambda_{j}+\mu
$$

whenever $\mathrm{I} \subseteq\{1, \ldots, n\}, 0 \leqq \lambda_{j} \leqq\left|\mathrm{E}_{j}\right|-r_{j}(1 \leqq j \leqq p), 0 \leqq \mu \leqq \sum_{1}^{p} s_{j}-n$. Write

$$
\mathrm{J}=\left\{j: 1 \leqq j \leqq p, \lambda_{j}=0\right\} \text {. }
$$

Then the above requirement is that

$$
\left|\mathrm{A}(\mathrm{I}) \cup \bigcup_{j \notin \mathrm{J}} \mathrm{E}_{j}\right|+\left|\bigcup_{j \notin J} \mathrm{D}_{j} \cup \mu\left(\mathrm{D}_{1} \cup \ldots \cup \mathrm{D}_{p}\right)\right| \geqq|\mathrm{I}|+\sum_{j \notin \mathrm{J}}\left(\left|\mathrm{E}_{j}\right|-r_{j}\right)+\mu
$$

whenever $\mathrm{I} \subseteq\{1, \ldots, n\}, \mathrm{J} \subseteq\{1, \ldots, p\}, 0 \leqq \mu \leqq \sum_{1}^{p} s_{j}-n$. This means that

$$
|\mathrm{A}(\mathrm{I}) \cap \mathrm{E}(\mathrm{J})|+\left|\bigcup_{j \neq \mathrm{J}} \mathrm{D}_{j} \cup \mu\left(\mathrm{D}_{1} \cup \ldots \cup \mathrm{D}_{p}\right)\right| \geqq|\mathrm{I}|-\sum_{j \neq \mathrm{J}} r_{j}+\mu
$$

whenever $\mathrm{I} \subseteq\{1, \ldots, n\}, \mathrm{J} \subseteq\{1, \ldots, p\}, 0 \leqq \mu \leqq \sum_{1}^{p} s_{j}-n$. 
For $\mu=0$, this condition becomes

i.e.

$$
|\mathrm{A}(\mathrm{I}) \cap \mathrm{E}(\mathrm{J})|+\sum_{j \neq \mathrm{J}}\left(s_{j}-r_{j}\right) \geqq|\mathrm{I}|-\sum_{j \neq \mathrm{J}} r_{j},
$$

$$
|\mathrm{A}(\mathrm{I}) \cap \mathrm{E}(\mathrm{J})| \geqq|\mathrm{I}|-\sum_{j \neq \mathrm{J}} s_{j} \text {, }
$$

for all $\mathrm{I} \subseteq\{1, \ldots, n\}, \mathrm{J} \subseteq\{1, \ldots, p\}$. Again, for $\mu>0$, condition (5) becomes

i.e.

$$
|\mathrm{A}(\mathrm{I}) \cap \mathrm{E}(\mathrm{J})|+\sum_{j=1}^{p}\left(s_{j}-j_{j}\right) \geqq|\mathrm{I}|-\sum_{j \notin \mathrm{J}} r_{j}+\sum_{j=1}^{p} s_{j}-n,
$$

e.

$$
|\mathrm{A}(\mathrm{I}) \cap \mathrm{E}(\mathrm{J})| \geqq|\mathrm{I}|-n+\sum_{j \in \mathrm{J}} r_{j}
$$

for all $\mathrm{I} \subseteq\{1, \ldots, n\}, \mathrm{J} \subseteq\{1, \ldots, p\}$. Thus $\mathfrak{A}$ has a transversal $\mathrm{T}$ subject to (1) if and only if (6) and (7) hold for all I, J, This implies our assertion.

\section{REFERENCES}

1. L. R. Ford, Jr, and D. R. Fulkerson, Flows in Networks (Princeton, 1962).

2. P. Hall, On representatives of subsets, J. London Math. Soc. 10 (1935), 26-30.

3. A. J. Hoffman and H. W. Kuhn, On systems of distinct representatives, Linear Inequalities and Related Systems, Annals of Mathematics Studies 38 (Princeton, 1956), 199-206.

4. L. Mirsky, Hall's criterion as a "self-refining" result, Monatsh. Math. 73 (1969), 139-146.

5. L. Mirsky, Transversal Theory (New York and London, 1971).

6. H. Perfect, Studies in Transversal Theory (Ph.D. Dissertation, University of London, 1969).

7. R. Rado, A theorem on independence relations, Quart. J. Math. Oxford Ser. 13 (1942), 83-89.

8. D. J. A. Welsh, Some applications of a theorem of Rado, Mathematika 15 (1968), 199-203.

\section{UNIVERSITY OF SHEFFIELD}

